\title{
MANANTIALES DE UNA PARTE DEL VALLE CENTRAL DE COSTA RICA
}

\author{
Asdrúbal Vargas \\ Instituto Costarricense de Electricidad \\ Centro Servicio Exploración Subterránea \\ E-Mail: avargass@ice.go.cr
}

\begin{abstract}
An inventory was made of springs in part of the Central Valley of Costa Rica. The springs with major discharge rates are Chorros (Tacares de Grecia), Puente de Mulas, La Libertad and Ojo de Agua. Many springs are used to supply potable water to the population of San José, Alajuela and Heredia. The total discharge of all springs can provide water for about 3 million people.

The major springs discharge groundwater from a volcanic aquifer formed by a fractured lava flow along the canyon of Virilla river and near to the town of Grecia. Many minor springs occurr to the south of Escazú and cerro Guarari, in Heredia.

Some are vulnerable to anthropogenic pollution. To reduce the risk of contamination this work discusses some methods to define protection zones. The analytical method is more precise in defining the recharge zone of a spring, but requieres too much information. The manual method can be modified to define a circular zone with the center located up to the spring.

RESUMEN: En este trabajo se ha realizado un inventario de los manantiales que están siendo utilizados para el abastecimiento de agua potable en las hojas topográficas Abra, Naranjo y Barba. La mayoría de los manantiales son de bajo caudal $(<10$ 1/s), pero existen mas de diez manantiales con caudales altos (> 100 1/s). Los manantiales de mayor descarga son los Chorros, Fuente Rosales, Ojo de Agua, La Libertad, Puente de Mulas; la mayoría de ellos asociados con acuíferos en lavas fisuradas.

Los manantiales de mayor caudal identificados en las hojas topográficas Abra y Naranjo, se encuentran rodeados de desarrollos urbanos, mientras que los manantiales de menor caudal se encuentran en zonas altas de la cordillera Volcánica Central o en las faldas de los cerros de Escazú. Estos resultados aumentan aún más la necesidad de contar con un servicio de alcantarillado sanitario adecuado para evitar la contaminación de los manantiales urbanos. Con un análisis de la demanda probable de agua se ha estimado que el caudal total de los manantiales inventariados es suficiente para abastecer una población de 3000000 de personas.

Considerando la importancia de proteger los manantiales que se encuentran rodeados de desarrollo urbano se propone utilizar un método matemático analítico para definir la zona de captura del agua subterránea de manantiales de mayor caudal. Este método se puede aplicar para acuíferos confinados o libres.
\end{abstract}

\section{INTRODUCCIÓN}

Ante la creciente demanda de agua los manantiales constituyen fuentes naturales de agua de buena calidad, que surgen a la superficie de manera natural, sin necesidad de bombeo, lo cual representa ahorro de energía y recursos eco- nómicos. Los manantiales representan descargas de agua subterránea que salen a la superficie del terreno, en el lugar donde el nivel de saturación del agua en el acuífero corta la superficie topográfica y se forman debido a cambios verticales u horizontales en la conductividad hidráulica de los materiales geológicos. Los manantiales de 
mayor caudal del Valle Central se presentan en materiales volcánicos como lavas, o brechas y entre otros se pueden mencionar Los Chorros en Tacares de Grecia, Ojo de Agua, La Libertad y Fuente Zamora con un caudal promedio superior a $100 \mathrm{l} / \mathrm{s}$.

Cada día aumenta la demanda de agua potable, siendo necesario llevar a cabo un inventario de los manantiales utilizados y del caudal de explotación promedio, como primer paso para la protección. Por lo tanto, el objetivo central de este trabajo consiste en identificar los principales manantiales de una parte del Valle Central, contenidos en las hojas topográficas Abra, Naranjo y Barva, escala 1: 50000 del Instituto Geográfico Nacional, donde se ubica gran parte de la población del Valle Central de Costa Rica (Fig. 1). Además, se pretende exponer las metodologías para definir el área de captura del agua subterránea de un manantial y consecuentemente la zona de protección. La zona de estudio está limitada al Norte por la cordillera Volcánica Central, al Este por los cerros de la Carpintera y al sur por los cerros de Escazú. La precipitación media anual varía de $2500 \mathrm{~mm}$ en las partes bajas hasta 3800 mm en las partes altas del volcán Poás. La hidrografía se caracteriza por un colector principal, el río Virilla, alimentado por numerosos afluentes en la parte Norte del valle.

Esta investigación se ha llevado a cabo por medio de un inventario de los manantiales que están en concesión según el departamento de

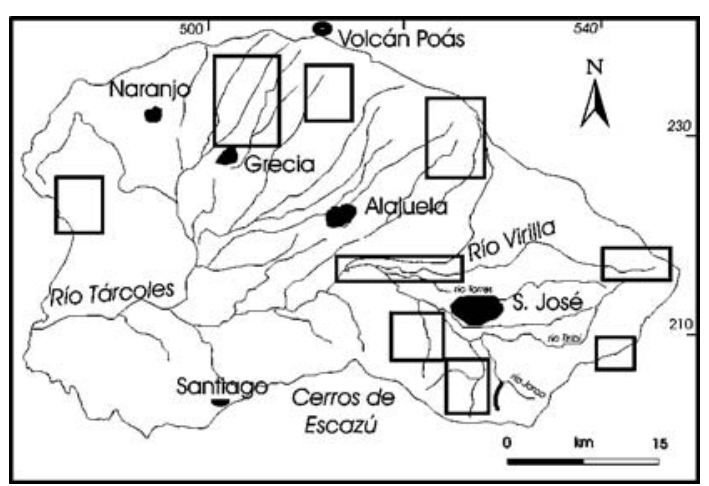

Fig. 1: Ubicación de la zona de estudio que comprende gran parte del Valle Central. Las zonas con mayor frecuencia de manantiales se encuentran marcadas por los rectángulos. aguas del Ministerio de Medio Ambiente (MINAE). Esta tarea se complementó con la búsqueda de información en el Instituto Costarricense de Acueductos y Alcantarillados (AyA) y en el Servicio Nacional de Aguas Subterráneas Riego y Avenamiento (SENARA).

Una vez recopilada la información se procedió a ubicar los manantiales sobre las hojas cartográficas y a preparar hojas electrónicas con la información de cada manantial inventariado. Posteriormente, se estudiaron varias metodologías de definición de zonas de captura de pozos y se aplicó una de ellas a los manantiales de mayor caudal identificados en cada hoja topográfica. Si bien las características físico-químicas del agua en los manantiales es un factor de gran importancia para definir su uso y protección, en este trabajo no se ha considerado la calidad química del agua, ya que se pretende realizar esta valoración en una investigación posterior.

La primera investigación sobre manantiales en el Valle Central fue realizada por Fernández (1968) como parte de un reconocimiento hidrogeológico en la cuenca de los ríos Poás, Ciruelas y Tibás. Losilla et al. (1992) realizaron un estudio biofísico y socioeconómico en el cerro Guararí, identificando varios manantiales y proponiendo medidas de protección para los mismos. Gómez (1987) evaluó el potencial de los manantiales y acuíferos en el sector de Puente de Mulas; Arredondo (1994 a y b) realizó un estudio sobre varios manantiales del cantón de San Antonio de Belén, delimitando las zonas de protección del manantial Ojo de Agua y Belén entre otros.

\section{CLASIFICACIÓN Y TIPOS DE MANANTIALES}

Las tres variables que definen la cantidad de agua de un manantial son la cantidad de precipitación, el área de recarga y la permeabilidad del terreno. Los manantiales se pueden clasificar de acuerdo a la descarga de agua, por medio del método de Meinzer, que distribuye los manantiales según la descarga de agua en ocho categorías, considerándose la primera para manantiales con caudales superiores a $2800 \mathrm{l} / \mathrm{s}$, mientras que en la 
octava categoría se encuentran aquellos con un caudal menor a 7,9 ml/s (Cuadro 1).

Cuadro 1

Clasificación de manantiales según descarga, método de Meinzer

\begin{tabular}{cc}
\hline Categoría & Descarga \\
\hline Primera & $>2,83 \mathrm{~m}^{3} / \mathrm{s}$ \\
Segunda & Entre 0,283 y $2,83 \mathrm{~m}^{3} / \mathrm{s}$ \\
Tercera & Entre 28,3 y $283 \mathrm{l} / \mathrm{s}$ \\
Cuarta & Entre 6,31 y $28,31 / \mathrm{s}$ \\
Quinta & Entre 0,631 y $6,31 \mathrm{l} / \mathrm{s}$ \\
Sexta & Entre 63,1 y $631 \mathrm{ml} / \mathrm{s}$ \\
Séptima & Entre 7,9 y $63,1 \mathrm{ml} / \mathrm{s}$ \\
Octava & $<$ de $7,9 \mathrm{ml} / \mathrm{s}$ \\
\hline
\end{tabular}

Fuente: Todd (1980).

Además, los manantiales se distinguen por las condiciones hidrogeológicas que dan lugar a la descarga de agua. La mayoría de manantiales se originan cuando la superficie de la zona saturada corta la superficie del terreno. Acuíferos formados por materiales permeables pueden originar manantiales cuando la superficie freática corta el talud de un valle (Fig. 2a).

Es frecuente dentro del área de estudio, que capas de piroclastos permeables (limitados por capas de menor permeabilidad) que reciben recarga suficiente contengan una zona saturada de agua cuya superficie corta la superficie del terreno en uno o varios puntos y originen manantiales por lo general de bajo caudal (Fig. 2b). Como ejemplo se mencionan los manantiales de la zona del Cerro Guararí, Sacramento, Lajas y Huacalillo en la hoja Barva.

Los abanicos coluvio-aluviales limitados por un basamento de menor permeabilidad pueden dar origen a manantiales (Fig. 2c). Ejemplos de estos manantiales se encuentran en las zonas de Bebedero y San Antonio de Escazú, al pie de los cerros de Escazú.

Otro tipo de manantial es el provocado por la presencia de una falla, que distorsiona el flujo de agua subterránea, lo que permite la surgencia de agua en forma de manantial. Ejemplos de éste tipo de manantial se observa en algunos
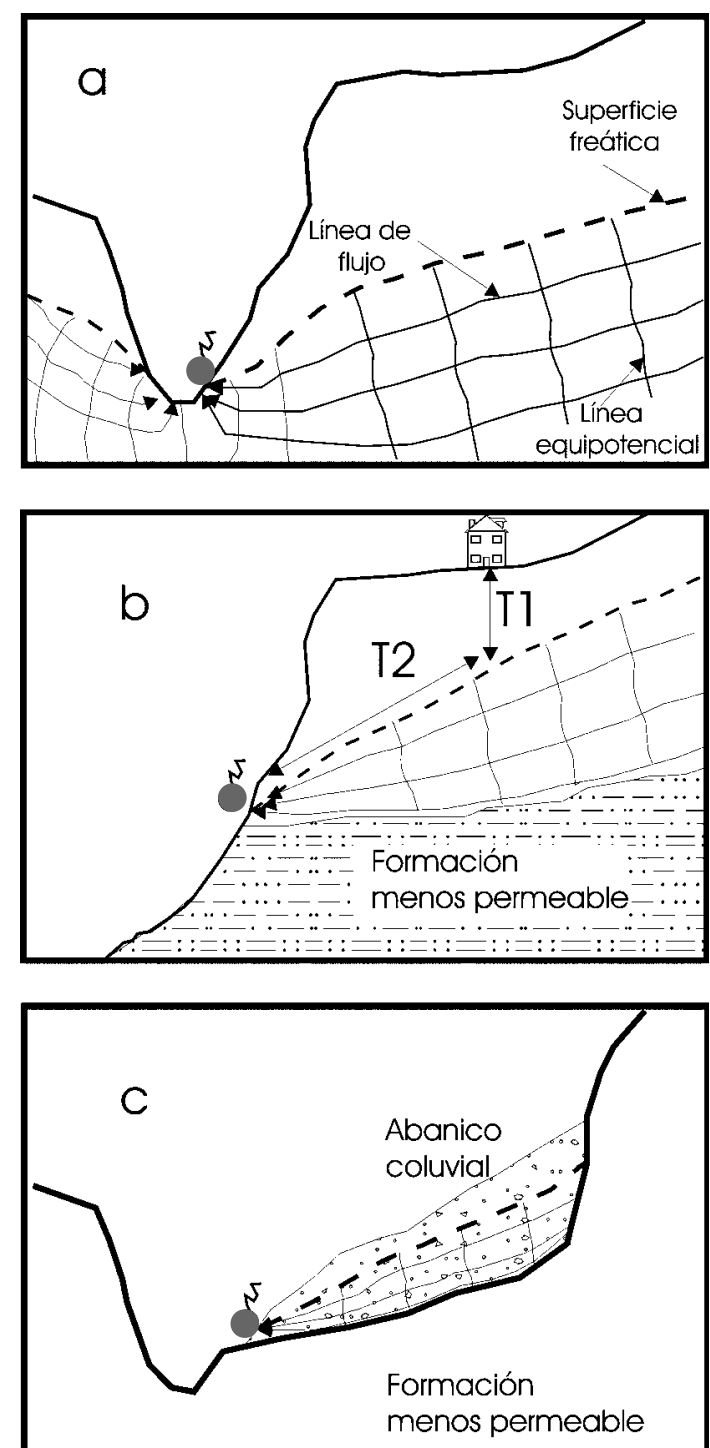

Fig. 2: Tipos de manantiales según condición hidrogeológica. a) Manantial en depresión. b) Manantial de contacto. c) Manantial en abanico coluvial.

tramos de la Falla de Alajuela, como en el sector de Tacares de Grecia.

Acuíferos formados en coladas de lava fracturadas y limitadas en la base por formaciones menos permeables pueden originar manantiales de gran caudal cuando la superficie freática corta la superficie (Ojo de Agua) o el talud de un cañón o valle (Fig. 3). Este tipo de manantiales se presentan en varios sectores del cañón del 


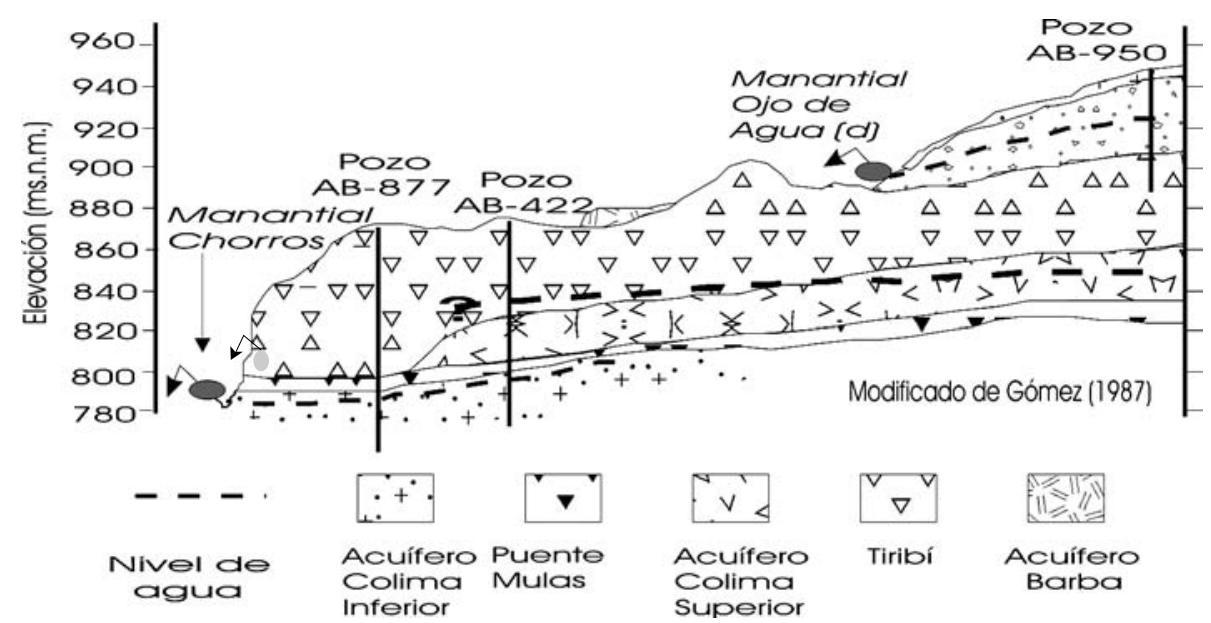

Fig. 3: Perfil hidrogeológico mostrando el origen de los manantiales Ojo de Agua y Chorros. En ambos casos existen unidades lávicas fracturadas limitadas por unidades menos permeables.

río Virilla, como por ejemplo en el sector de Puente de Mulas.

\section{MANANTIALES DE UNA PARTE DEL VALLE CENTRAL}

El caudal total de los manantiales inventariados dentro del Valle Central que se encuentran en concesión es de 5543 1/s (Cuadro 2). Se estima que con una dotación de 150 litros por persona por día, la cantidad de agua producida por estos manantiales puede suplir una población de 3 millones de habitantes. La dotación utilizada es sugerida por AyA (2000) para acueductos rurales. Sin embargo, se considera aquí que este valor es adecuado para comunidades urbanas y semiurbanas.
Comparando las hojas topográficas entre sí, es posible observar que en el caso de la hoja Barva, aunque el número de manantiales es menor que en la hoja Naranjo, las descargas de agua son mayores. En la hoja Abra se contabilizó el menor número de manantiales pero las descargas superan las registradas en la hoja Naranjo.

Es importante notar que aunque en la hoja Naranjo se presentan más de 280 manantiales, estos suman un caudal de 1279 1/s, en la hoja Barva, que contiene un número menor de manantiales, el caudal es mucho mayor, casi 2549 1/s. La ocurrencia y caudal de los manantiales dentro del Valle Central está muy relacionada con la precipitación, ya que en las partes altas se presentan los valores más elevados de precipitación, la cual se infiltra y pasa a formar parte del agua subterránea

Cuadro 2

Manantiales en concesión por hoja topográfica

\begin{tabular}{cccc}
\hline Hoja topográfica & \# manantiales en concesión & Caudal total (1/s) & Abastecimiento (\# personas) \\
\hline Naranjo & 284 & 1279,1 & 736762 \\
Abra & 128 & 1715,4 & 988070 \\
Barba & 201 & 2549,1 & 1468282 \\
\hline Total & 613 & 5543,6 & 3193114 \\
\hline
\end{tabular}

Fuente: Departamento de Aguas, MINAE. (2000). 
almacenada que luego es descargada en los manantiales. De aquí se desprende el hecho que en la hoja Barva y Naranjo existe un potencial de agua subterránea muy importante definido por abundante precipitación, materiales de adecuada permeabilidad y capacidad de infiltración. En la hoja Abra se presentan manantiales de gran caudal en el cañón del río Virilla, pero la zona de recarga está fuera de esta hoja cartográfica.

Los manantiales de mayor caudal son los denominados Los Chorros, en Tacares de Grecia, con un caudal de descarga de casi 1000 l/s, seguido por el manantial Chorros en San Antonio de Belén, Puente de Mulas y Ojo de Agua con caudales cercanos a los $450 \mathrm{l} / \mathrm{s}, 700 \mathrm{l} / \mathrm{s}$ y $300 \mathrm{l} / \mathrm{s}$ respectivamente.

\section{Manantiales en la hoja Abra}

Si bien gran parte del área comprendida dentro de la hoja topográfica corresponde a zonas urbanas, es posible señalar dos zonas con presencia de manantiales de gran caudal. A lo largo del río Virilla, entre San Juan de Tibás y San Antonio de Belén, se presentan descargas de agua subterránea provenientes del acuífero Colima y denominados La Libertad, Valencia, Ánimas, Fuente Zamora, Puente de Mulas, Freddy, Chorros y Penal (Fig. 4). Varios de estos manantiales se constituyen como fuentes de abastecimiento de agua potable de AyA para la Gran Área Metropolitana, ya que aportan caudales que varían de 100 a 1000 1/s, constituyéndose en fuentes de gran importancia (Cuadro 3).

Además, cerca de San Antonio de Belén (218000-516000) se presentan varios manantiales de gran caudal que descargan agua subterránea proveniente del acuífero Barva que se encuentra subyacido por una secuencia de materiales de permeabilidad variable (Fig. 3). El contenido de isótopos estables provenientes de estos manantiales, como por ejemplo Ojo de Agua y Echeverría, indican que estos manantiales tienen su recarga a una altura superior a los $2000 \mathrm{~m}$ s.n.m., desde ríos influentes que, a su vez, se alimentan de escorrentía en las partes altas (Darling, et. al., 1984). Esta recarga proveniente de ríos se

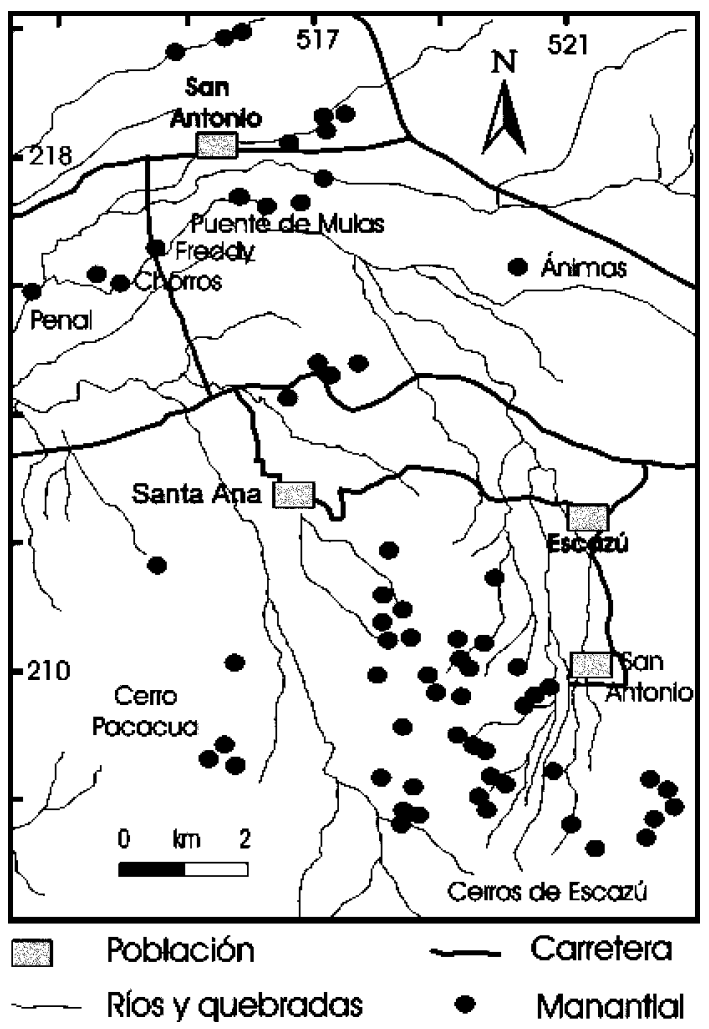

Fig. 4: Ubicación de los manantiales en parte de la hoja cartográfica Abra. Los puntos negros representan manantiales.

Cuadro 3

Manantiales de mayor caudal en la hoja Abra

\begin{tabular}{llll}
\hline $\begin{array}{l}\text { Nombre } \\
\text { del manantial }\end{array}$ & Ubicación & $\begin{array}{l}\text { Caudal } \\
\text { medio }(1 / \mathrm{s})\end{array}$ & $\begin{array}{l}\text { Tipo de } \\
\text { roca }\end{array}$ \\
\hline
\end{tabular}

\begin{tabular}{|c|c|c|c|}
\hline Freddy- Chorros & $\begin{array}{l}\text { San Antonio, } \\
\text { Belén }\end{array}$ & 1000,0 & $\begin{array}{l}\text { Lavas Fm. } \\
\text { Colima Sup. }\end{array}$ \\
\hline Puente de Mulas & $\begin{array}{l}\text { San Antonio, } \\
\text { Belén }\end{array}$ & 360,0 & $\begin{array}{l}\text { Lavas } \\
\text { Fm. Colima }\end{array}$ \\
\hline $\begin{array}{l}\text { Fuente } \\
\text { Potrerillos }\end{array}$ & San Rafael & 193,2 & $\begin{array}{l}\text { Lavas Fm } \\
\text { Colima Inf. }\end{array}$ \\
\hline Echeverría & $\begin{array}{l}\text { Flores, } \\
\text { Heredia }\end{array}$ & 180,0 & $\begin{array}{l}\text { Lavas Fm. } \\
\text { Barba }\end{array}$ \\
\hline $\begin{array}{l}\text { Fuente Tajo } \\
\text { Zamora }\end{array}$ & $\begin{array}{l}\text { San Antonio, } \\
\text { Belén }\end{array}$ & 123,6 & $\begin{array}{l}\text { Lavas } \\
\text { Fm. Colima Sup }\end{array}$ \\
\hline Ánimas & $\begin{array}{l}\text { Barreal, } \\
\text { Heredia }\end{array}$ & 100,0 & $\begin{array}{l}\text { Lavas } \\
\text { Fm. Colima Sup. }\end{array}$ \\
\hline La Libertad & $\begin{array}{l}\text { Sto. Domingo, } \\
\text { Heredia }\end{array}$ & 100,0 & $\begin{array}{l}\text { Lavas } \\
\text { Fm. Colima }\end{array}$ \\
\hline
\end{tabular}

Total 2160

Fuente: Departamento de estudios básicos, AyA. 
podría mezclar con una porción pequeña de infiltración directa de la lluvia.

Al Sur de Escazú se presentan numerosas concesiones que incluyen manantiales para abastecer las comunidades de Bebedero, San Antonio y Chiverral por medio de acueductos rurales. Estos manantiales están protegidos por la Zona Protectora de los Cerros de Escazú, sin embargo es conveniente fortalecer las medidas de protección de las captaciones.

\section{Manantiales en la hoja Naranjo}

Se identifican dos zonas con numerosas fuentes de agua subterránea. Una de estas zonas se encuentra compuesta por las cuencas de los ríos Prendas, Rosales, Sarchí y la parte alta del río Colorado. En estas cuencas se presentan manifestaciones importantes como los Chorros (> 900 1/s), Fuente Rosales, Fuente Amelia, Fuente Patal, y Fuente Los Ángeles. El gran potencial de agua subterránea está favorecido por materiales lávicos y piroclastos de gran permeabilidad, abundante precipitación anual y una pendiente pronunciada. Gran parte de la zona de recarga se encuentra en la reserva forestal Grecia.

Las fuentes de mayor caudal son Los Chorros, Fuentes Patal, Fuentes Rosales, Fuentes Amelia, Fuentes Municipales de Grecia, Fuentes Los Ángeles y Fuentes Victoria que se ubican alrededor de Grecia (Fig. 5). La descarga de agua subterránea en el sector de los Chorros ocurre solo en la margen izquierda del río Prendas, incluyendo las descargas en la quebrada Zamora. Su origen se debe a las características del acuífero en cuanto a porosidad primaria en forma de vacuolas y porosidad secundaria por fracturas; además, este acuífero se encuentra confinado por una capa de baja permeabilidad en la base que se suma al efecto de plegamiento de los materiales que ha originado una zona deprimida donde descarga el agua subterránea. Los mayores caudales de descarga varían de 1000 l/s hasta 15 l/s, aunque el caudal mayor alcanza en determinados períodos hasta 1600 1/s para el caso de Los Chorros (Cuadro 4). Considerando que el caudal total de estos manantiales mayores alcanza 1770,5 1/s, se estima

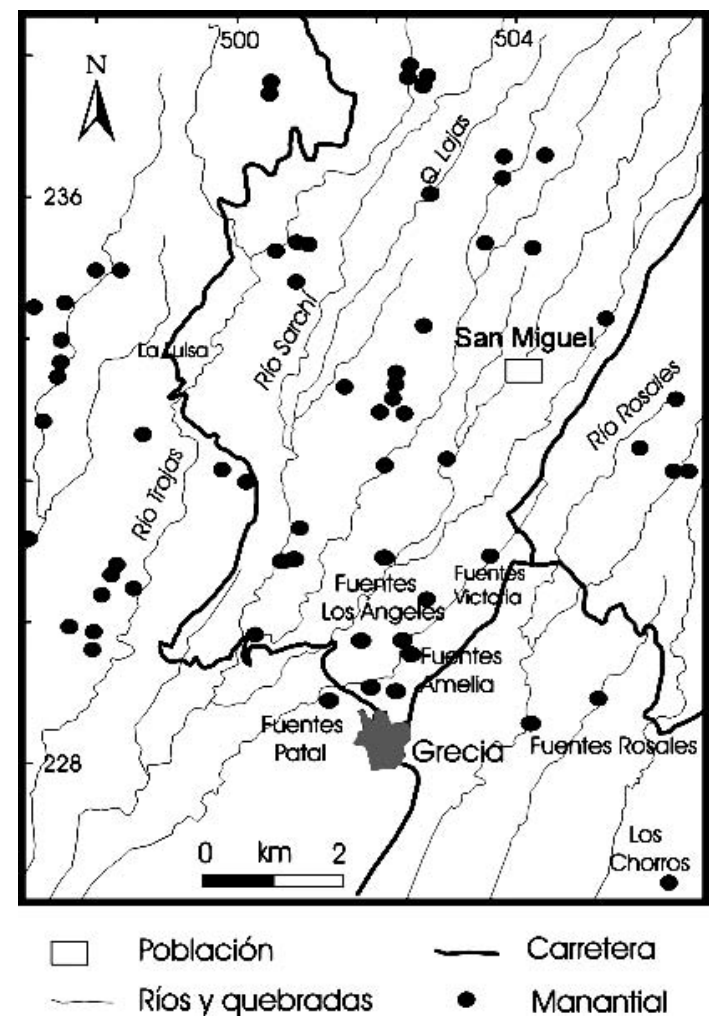

Fig. 5: Ubicación de los manantiales en parte de la hoja cartográfica Naranjo. Los puntos negros representan manantiales.

que con una dotación de 150 l/p/d, se podría abastecer una población de un millón de personas.

El sector de los Chorros se encuentra dentro de un pliegue de propagación de falla, con dos limbos que controlan la descarga del agua subterránea, originada como recarga en las partes altas del volcán Poás. Aunque en el limbo sur del pliegue y en el frente del escarpe de la falla de Alajuela hay surgencias de agua subterránea, estas son de menor caudal. Sin duda los rasgos estructurales del área controlan la descarga del agua subterránea.

Según información obtenida del Instituto Costarricense de Acueductos y Alcantarillados (AyA), con el caudal de los manantiales Los Chorros se logra abastecer a la comunidad de Atenas, Tacares, Bodegas, Finca La Argentina, Santa Eulalia y Barrio Mercedes, entre otras. Un inventario preliminar de manantiales en el área 
Cuadro 4

Caudales de los principales manantiales hoja Naranjo

\begin{tabular}{|c|c|c|c|c|c|}
\hline Manantial & Latitud & Longitud & Qmedio (1/s) & Qmayor 1/s) & Qmenor $(1 / \mathrm{s})$ \\
\hline Los Chorros & 226100 & 505900 & 999.4 & 1644.0 & 498.0 \\
\hline Fte. Patal (Rebose) & 228850 & 501300 & 290.1 & 396.0 & 143.0 \\
\hline Fte. Rosales & 228500 & 504200 & 277.1 & 426.0 & 215.0 \\
\hline Fte. Amelia (Rebose) & 229700 & 502400 & 117.6 & 261.0 & 43.0 \\
\hline Ftes. Municipales Grecia & 231500 & 500950 & 38.5 & 56.0 & 21.0 \\
\hline Fte. Los Angeles & 230900 & 502100 & 32.1 & 46.0 & 25.0 \\
\hline Fte. Victoria & 230900 & 503600 & 15.7 & 196.0 & 3.0 \\
\hline
\end{tabular}

Fuente: Departamento Estudios Básicos AyA

$\mathrm{Q}_{\text {medio }}$ : caudal promedio de descarga. $\mathrm{Q}_{\text {mayor }}$ : caudal máximo registrado. $\mathrm{Q}_{\text {menor }}$ : caudal mínimo registrado

de estudio utilizando el registro del departamento de aguas del Ministerio de Ambiente y Energía (MINAE) indica que cerca del $80 \%$ de los manantiales tienen caudales que oscilan entre 0,1 hasta 10 l/s.

Al sur de Palmares se presenta otra zona con gran número de fuentes de agua, con caudales más pequeños que en la zona anteriormente señalada. Esto se debe a materiales volcánicos de menor permeabilidad y a una recarga más reducida. Estos manantiales abastecen poblados como Santiago, Rincón de Mora y Berlín. En total, para la hoja Naranjo se contabilizaron 284 concesiones de agua en manantiales para un total de 1279,1 1/s sin tomar en cuenta, aquellos captados por el AyA, que representan un volumen muy importante.

En el sector Noreste de la hoja Naranjo, donde ocurren los manantiales de mayor caudal, se han identificado cuatro unidades hidrogeológicas principales denominadas lavas inferiores, asociadas con la unidad Colima, unidad de ignimbritas asociadas con la unidad Tiribí (Echandi, 1981), unidad de lavas Poás y la unidad de tobas. Según Mora (1995) por la presencia de vacuolas, el intenso fracturamiento y por la presencia de brechas principalmente en su parte superior, la unidad Colima tiene una alta capacidad de transmitir agua y en determinadas condiciones de almacenamiento de la misma. Esta unidad aflora en el río Tacares en las coordenadas 226500 y 505500 , mientras que en el río Prendas en las coordenadas 226300 y 505900 . En este úl- timo punto se origina una descarga del agua subterránea que da origen a los manantiales denominados Los Chorros.

La unidad de ignimbritas, tienen menor permeabilidad, sin embargo, en los sectores fracturados se aumenta la capacidad de transmitir agua. Por su parte, la unidad de lavas Poás presenta una alta permeabilidad. Desde esta unidad se originan numerosos manantiales, entre ellos los manantiales denominados Poasito, La Choyotera y Guatusa. Las unidades de tobas presentan permeabilidades de baja a muy baja, siendo captadas por los pozos en sectores arenosos.

\section{Manantiales en la hoja Barva}

Los manantiales en la hoja Barva se encuentran localizados alrededor de Huacalillo, San Miguel, Roble y Sacramento (Fig. 6). Gran número de estos manantiales descargan hacia el río Ciruelas. Algunos de ellos corresponden a las descargas de agua subterránea contenida en materiales piroclásticos asociados con el volcán Barva. Es probable que la recarga de estos manantiales de gran altitud tengan su origen en la precipitación que se presenta en las partes altas. Si bien dentro de esta hoja existen numerosos manantiales, la mayoría de ellos son de bajo caudal. La zona comprendida dentro de esta hoja cumple una función de recarga de acuíferos profundos por medio de infiltración en los lechos de 


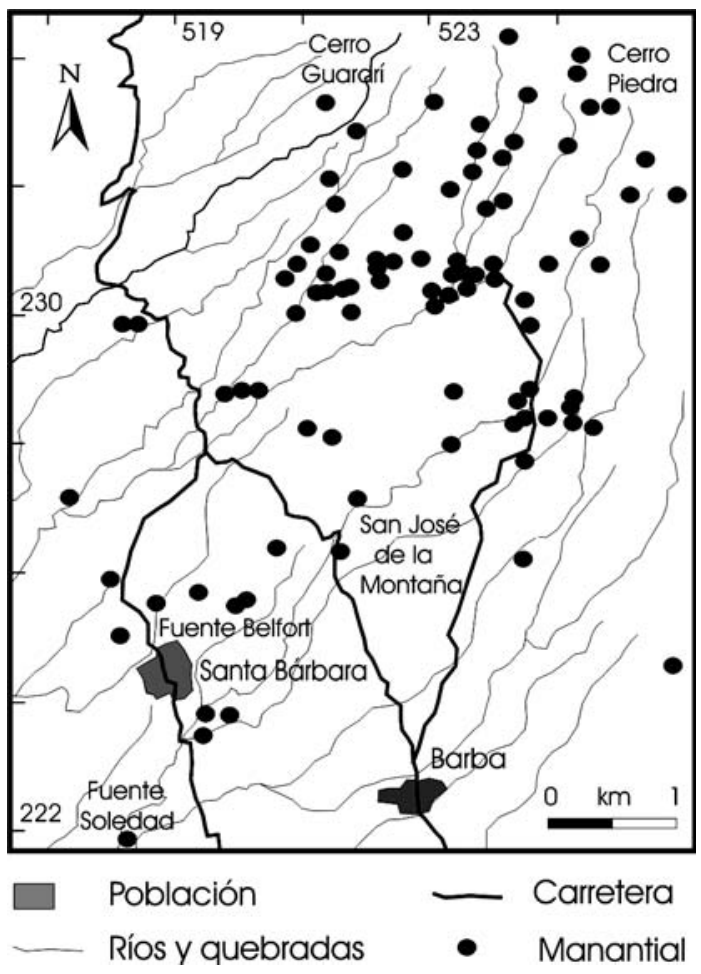

Fig. 6: Ubicación de los manantiales en parte de la hoja cartográfica Barva. Los puntos negros representan manantiales.

los ríos donde afloran las lavas fracturadas constituyentes de los acuíferos Barva, La Libertad y Colima Superior. Algunos de los manantiales son utilizados para abastecer acueductos administrados por AyA, como son fuente Los Zamora, La Soledad, Santa Bárbara y Belfort (Cuadro 5).

\section{Magnitud de los manantiales}

En el área de estudio predominan manantiales de magnitudes 5 y 6 , es decir, son manantiales con caudales entre 0,1 y 10 l/s. No existen manantiales de las categorías 1 y 2. En la hoja Abra se presenta el mayor número de manantiales de mayor orden (Cuadro 6). Esto se debe a la descarga que origina el acuífero Colima en varios puntos donde afloran sus miembros La Libertad, Colima Inferior y Superior.

\section{Cuadro 6}

Clasificación de los manantiales según escala de Meinzer

\begin{tabular}{cccc}
\hline Magnitud & H. Abra & H. Barba & H. Naranjo \\
\hline 1 & 0 & 0 & 0 \\
2 & 2 & 0 & 1 \\
3 & 6 & 7 & 1 \\
4 & 7 & 30 & 17 \\
5 & 20 & 85 & 77 \\
6 & 69 & 65 & 143 \\
7 & 26 & 8 & 46 \\
8 & 2 & 6 & 0 \\
\hline
\end{tabular}

\section{ÁREAS DE CAPTURA DE MANANTIALES}

El área de captura de un manantial se puede determinar por medio de consideraciones hidrogeológicas. Esta se define como el área geográfica donde se infiltra agua que se dirige hacia el punto de descarga o manantial. A partir de esta zona de captura es posible definir un área de protección. Para evitar la amenaza de contaminación

Cuadro 5

Caudales de los principales manantiales hoja Barba

\begin{tabular}{lccccc}
\hline Manantial & Latitud & Longitud & $\mathrm{Q}_{\text {medio }}(1 / \mathrm{s})$ & $\mathrm{Q}_{\text {mayor }}(1 / \mathrm{s})$ & $\mathrm{Q}_{\text {menor }}(1 / \mathrm{s})$ \\
\hline Fuente Belfort & 225500 & 518700 & 6,7 & 13,0 & 2,5 \\
Fuente Santa Bárbara & 223980 & 518820 & 7,6 & 8,0 & 6,9 \\
Fuentes Soledad & 221900 & 518160 & 19,3 & 31,1 & 10,0 \\
Fuentes Zamora & 218280 & 516620 & 27,6 & 297,0 & 3,0 \\
\hline
\end{tabular}

Fuente: Departamento Estudios Básicos AyA.

$\mathrm{Q}_{\text {medio }}$ : caudal promedio de descarga. $\mathrm{Q}_{\text {mayor }}$ : caudal máximo registrado. $\mathrm{Q}_{\text {menor }}$ : caudal mínimo registrado 
del agua toda actividad potencialmente contaminante debe ser controlada o limitada a un nivel adecuado dentro de esta zona de protección. Sin embargo, debido a cuestiones socioeconómicas esta limitación resulta difícil de cumplir en su sentido amplio, por lo tanto, en la práctica se opta por realizar una división de la zona de captación en zonas menores para aplicar restricciones más severas en áreas cercanas al manantial. Esta subdivisión se puede realizar tomando en cuenta el tiempo de flujo horizontal, la distancia del manantial a la fuente contaminante y el tiempo de tránsito de un contaminante determinado.

Las zonas de subdivisión se pueden denominar área de protección máxima, área de protección interior y área de protección exterior. El área de protección máxima comprende un área pequeña alrededor del manantial. En esta área no se deberían permitir actividades que no estén relacionadas con la extracción del agua, e incluso estas actividades deberán ser rigurosamente controladas. La dimensión de esta área es por lo común arbitraria en términos legales. El área de protección interna tiene la finalidad de evitar la contaminación por fuentes antropogénicas y se puede establecer sus dimensiones basado en el tiempo de flujo horizontal (100 a 500 días) y en la densidad de población. El área de protección externa se puede delimitar en función de tiempo de flujo horizontal mayor que para las zonas anteriores, por ejemplo mayor a 500 días.

\section{Métodos para delimitar la zona de captura}

La delimitación de las zonas de captura puede basarse en diversos parámetros dependiendo de la amenaza de contaminación percibida que involucra: distancia horizontal, tiempo de flujo horizontal, proporción del área de recarga, dilución de contaminantes en la zona saturada o la capacidad de atenuación. No obstante, es común emplear una combinación del tiempo de tránsito en sentido vertical en la zona no saturada y cuasi horizontal en la saturada. Los métodos para delinear la zona de captura y, posteriormente con las precauciones del caso, la zona de protección se pueden dividir en manuales, semianalíticos y numéricos.

\section{Métodos manuales}

Los métodos manuales se consideran los más sencillos de aplicar. El método del radio fijo arbitrario involucra dibujar un círculo de radio específico alrededor de cada manantial. Aunque es un método muy sencillo debe ser tomado solo como una medida temporal hasta delimitar la zona con más detalle. Este método es muy útil en casos donde se requiere dar una atención inmediata ante una contaminación inminente. En Costa Rica la Ley de Aguas de 1942, que aún está vigente, en su artículo 31 declara como reserva a favor de la Nación, las tierras que circunden los sitios de captación o tomas surtidoras de agua potable, en un perímetro no menor de doscientos metros de radio y la zona forestal que protege el conjunto de terrenos en que se produce la infiltración de aguas potables, así como el de los que dan asiento a cuencas hidrográficas y márgenes de depósito, fuentes surtidoras o curso permanente de aguas. En el artículo 149 se establece la prohibición de destruir, tanto en los bosques nacionales como privados, los árboles situados a menos de sesenta metros de los manantiales que nazcan en cerros o a menos de cincuenta metros de los que nazcan en terrenos planos.

En el artículo 33 del capítulo IV de la ley forestal se señala como área de protección las áreas que bordean nacientes permanentes, definidas en un radio de cien metros medidos de modo horizontal. Además, las áreas de recarga y los acuíferos de los manantiales, cuyos límites serán determinados por los órganos competentes establecidos.

Losilla (1992) propone una metodología que considera aspectos hidrogeomorfológicos, al definir el área de protección con base en las divisorias de la cuenca hidrológica donde se encuentra el manantial. Se asume que los límites de la cuenca hidrogeológica coinciden con los límites de la cuenca hidrológica, según la cual se sigue la curva de elevación topográfica más baja del manantial, a ambos lados del mismo, hasta cruzar un río importante, considerado como influente; a partir de esto se sigue por las divisorias de las subcuencas hasta unirse en su parte superior. Esta metodología está diseñada para delimitar zonas de recarga de manantiales que son originados desde acuíferos someros. 
El método del radio fijo permite calcular la zona de captura de un manantial con recarga anual por medio de la siguiente expresión:

$$
A=\frac{q}{R}
$$

Donde:

A: Área de la zona de captura o de recarga $\left(\mathrm{m}^{2}\right)$

$\mathrm{q}$ : volumen anual autorizado $\left(\mathrm{m}^{3}\right)$

$\mathrm{R}$ : recarga anual (m)

Todd (1980) presenta una metodología similar a la anterior, pero que se puede utilizar por medio de un gráfico (Fig. 7), donde se relaciona la descarga, la recarga media anual y el área de la zona de recarga de un manantial. Se debería usar para tener una idea del área de recarga.

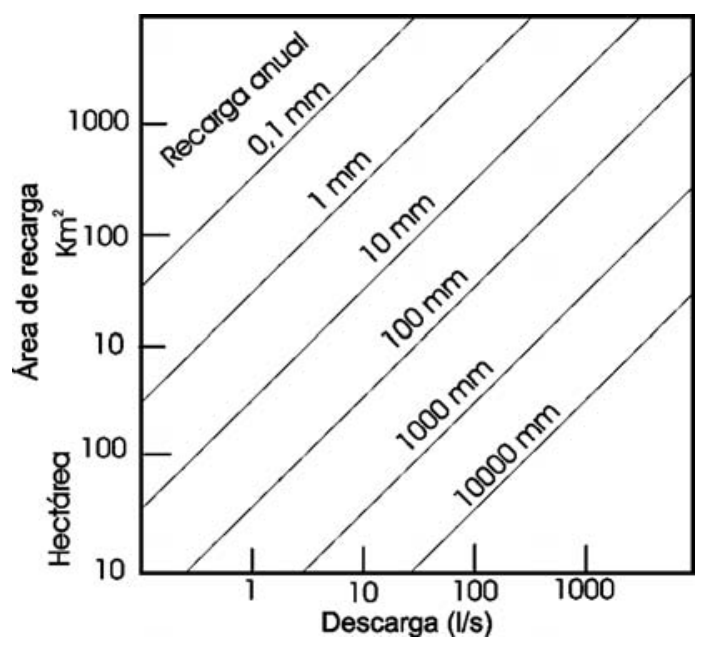

Fig. 7: Diagrama de Todd, que muestra la relación de la descarga media del manantial, la recarga media anual y el área de captura.

Además, es posible calcular el área de protección relacionada con un tiempo de viaje. En éste caso se puede aplicar la expresión:

$$
A=\frac{q \bullet t}{b \cdot n}=\left(\pi \cdot r^{2}\right)
$$

Donde:

$\mathrm{q}$ : volumen anual $\left(\mathrm{m}^{3} / \mathrm{d}\right)$

$\mathrm{t}$ : tiempo de tránsito (días)

b: espesor del acuífero (m) n: porosidad efectiva del acuífero (-)

r: radio de la zona de protección (m)

Otra formulación que permite calcular una zona de captura en forma radial involucra un tiempo de tránsito considerando tanto a la zona no saturada como a la saturada. Tomándose la zona no saturada como aquella comprendida entre la superficie del terreno y el nivel freático. El tiempo de tránsito en la zona no saturada se puede estimar por medio de la siguiente fórmula:

$$
t=\frac{B \cdot \theta}{k}
$$

mientras que para la zona saturada es la siguiente:

$$
t=\frac{d \bullet k \bullet i}{\theta}
$$

Donde :

B: espesor de la zona no saturada (m)

$\theta$ : porosidad efectiva

$\mathrm{k}$ : conductividad hidráulica $(\mathrm{m} / \mathrm{d})$

i : gradiente hidráulico $(\mathrm{m} / \mathrm{m})$

$\mathrm{d}$ : distancia (m)

t: tiempo (días)

En éste método se estima un tiempo de tránsito total estimado como la suma del tiempo en la zona no saturada (Fig. 2 b) denominada T1 y un tiempo de tránsito para la zona saturada (T2). Arredondo (1994a) define para el manantial Ojo de Agua una zona denominada $1 \mathrm{de} 250 \mathrm{~m}$ de radio tomando en cuenta un tiempo de tránsito total entre la zona no saturada y saturada de 100 días. Para esto consideró una porosidad efectiva de 0,1 , una conductividad hidráulica de $10 \mathrm{~m} / \mathrm{d}$ del acuífero Barva y un gradiente hidráulico de 0,025 . Este método debería complementarse con un análisis de substancias químicas en diferentes tipos de materiales de la zona no saturada.

\section{Método analítico}

La delineación de las zonas de captura de un manantial se puede lograr también por medio de la modificación de métodos de delimitación de zonas de captura de pozos con modelos 
semianalíticos. Esta metodología se encuentra descrita en Todd (1980), Fetter (1994) y en McWhorter \& Sunada (1999) y contempla varias expresiones matemáticas que se aplican dependiendo del régimen del acuífero. En el caso de acuíferos confinados se fundamenta en la expresión siguiente:

$$
x=\frac{-y}{\tan \left[\frac{2 \cdot \pi \cdot k \cdot b \cdot i \cdot y}{Q}\right]}
$$

$\mathrm{O}$, de acuíferos libres

$$
x=\frac{-y}{\tan \left\lceil\frac{\pi \cdot k \cdot\left(h_{1}^{2}-h_{2}^{2}\right) \cdot y}{Q \cdot L}\right\rceil}
$$

Donde:

y: valor de la coordenada en el eje y en un sistema de coordenadas cartesianas

$\mathrm{x}$ : valor de la coordenada en el eje $\mathrm{x}$ en un sistema de coordenadas cartesianas

$\mathrm{k}$ : conductividad hidráulica del medio geológico $(\mathrm{m} / \mathrm{d})$

b: espesor del acuífero $(\mathrm{m})$

q: Caudal $\left(\mathrm{m}^{3} / \mathrm{d}\right)$

1: Longitud entre dos puntos de observación del nivel freático $(\mathrm{m})$

$\mathrm{h}_{1}$ : elevación mayor del nivel freático $(\mathrm{m})$

$\mathrm{h}_{2}$ : elevación menor del nivel freático $(\mathrm{m})$
El ancho máximo de la zona de captura se determina según un acuífero confinado o libre respectivamente.

$$
\begin{aligned}
& y_{\text {max }}= \pm \frac{Q}{(2 \cdot k \cdot b \cdot i)} \\
& y_{\text {max }}= \pm \frac{Q \cdot L}{k \cdot\left(h_{1}^{2}-h_{2}^{2}\right)}
\end{aligned}
$$

Mientras que el punto de no retorno aguas abajo del manantial se determinaría según acuífero confinado o libre como:

$$
x_{o}=\frac{-Q}{(2 \cdot \pi \cdot k \cdot b \cdot i)}
$$

$$
x_{o}=\frac{-Q \cdot L}{\left[\pi \cdot k \cdot\left(h_{1}^{2}-h_{2}^{2}\right)\right]}
$$

Utilizando dichas expresiones se ha calculado el ancho máximo de cada zona de captura para los manantiales de mayor caudal (Cuadro 7).

Esta metodología está diseñada para definir las zonas de captura de pozos y debe complementarse con la definición de zonas de protección internas con los tiempos de tránsito de agentes contaminantes. Actualmente existen programas de cómputo que resuelven estas ecuaciones de

Cuadro 7

Cálculo de extensión lateral de las zonas de captura de manantiales de mayor caudal

\begin{tabular}{cccccc}
\hline Manantial & Acuífero & $\mathrm{Q}(\mathrm{m} 3 / \mathrm{d})$ & $\mathrm{T}(\mathrm{m} 2 / \mathrm{d})$ & $\mathrm{i}(\mathrm{m} / \mathrm{m})$ & $\mathrm{Ymax}(\mathrm{m})$ \\
\hline Echeverría & Barba & 15552 & 400 & 0,037 & 222,0 \\
Ojo de Agua & Barba & 25920 & 400 & 0,037 & 36,6 \\
Tajo Zamora & Colima Superior & 10674,7 & 5000 & 0,033 & 64,8 \\
Guachipelín & Colima Superior & 17280 & 5000 & 0,033 & 104,8 \\
Chorros & Colima Inferior & 8640 & 4500 & 0,015 & 128,0 \\
Potrerillos & Colima Inferior & 16675 & 4500 & 0,015 & 247,0 \\
La Libertad & La Libertad & 8640 & 1000 & 0,03 & 288,0 \\
Puente Mulas & Colima Superior & 64800 & 5000 & 0,033 & 392,8 \\
Chorros-Tacares & Colima Superior & 86400 & 5000 & 0,033 & 523,6 \\
\hline
\end{tabular}

Q: caudal medio; T: transmisividad; i: gradiente hidráulico; Ymax: ancho máximo de la zona de captura 
manera muy expedita y brindan una representación gráfica de la zona de captura y de las zonas de protección basadas en tiempos de tránsito. Uno de estos programas es el WHPA, que fue desarrollado por Blandford, Huyakorn \& Shu Wu (1993) del Centro Internacional de Modelaje de Aguas subterránea para ser utilizado por la Agencia de Protección Ambiental de los Estados Unidos (USEPA).

Una vez definida la zona de captura y las zonas internas por medio de los tiempos de tránsito se tendría una representación gráfica vista en planta como se muestra en la figura 8. Con éste método se incluye una zona de protección de la captación, definida entre el manantial y el punto de no retorno, ya que como se verá más adelante, el flujo de agua que llega al manantial desde esta zona es muy reducido comparada con la zona aguas arriba. Además, se definen las zonas de protección con base en tiempos de tránsito.

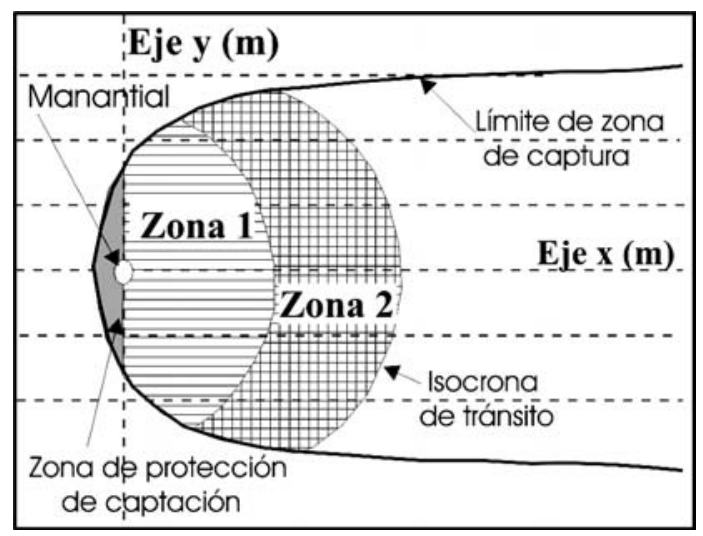

Fig. 8: Zona de captura diseñada con el método matemático analítico modificada para un manantial.

\section{Método numérico}

La estimación del área de captura de un manantial es posible llevarla a cabo también por medio de un modelo de funcionamiento hidráulico de un acuífero por métodos que resuelven la ecuación de Laplace de manera numérica. Estos métodos tienen la ventaja sobre los métodos analíticos que pueden simular condiciones de heterogeneidad del medio rocoso y varias condiciones hidrogeológicas como manantiales, no obstante, asumen que el medio es poroso pero no fracturado. Actualmente la construcción de modelos numéricos se puede realizar por medio de códigos de computadora que resuelven un gran número de ecuaciones requeridas en las celdas en que se ha discretizado el acuífero de manera muy rápida, como por ejemplo MODFLOW o ASTM.

Como ejemplo de aplicación se simuló la zona de captura de un manantial en un acuífero libre hipotético (Fig. 9). El acuífero tiene una conductividad hidráulica de $23 \mathrm{~m} / \mathrm{d}$ y un rendimiento específico (Sy) de 0,2. El acuífero se discretizó en 53 filas y en 56 columnas de ancho

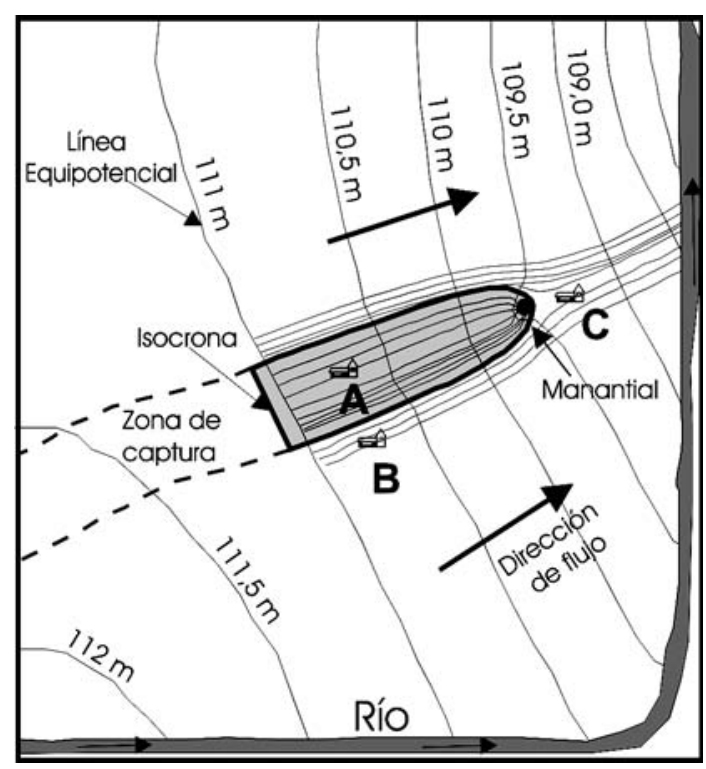

Fig. 9: Zona de captura de un manantial diseñada por medio de un modelo matemático numérico.

variable, de tal manera que se obtuviera mayor precisión en el centro del acuífero para simular el manantial. Se asignaron condiciones de frontera tipo río en el límite inferior y derecho y de no flujo en los límites restantes. Se corrió el modelo en estado estacionario para definir la superficie freática del acuífero. Posteriormente se definió una celda como tipo drenaje con una elevación de $109 \mathrm{~m}$ sobre el nivel de referencia.

A partir de éste modelo es posible establecer algunas consideraciones sobre la geometría de la zona de captura y el efecto de la ubicación de actividades potencialmente contaminantes. Por un lado el modelo numérico permite observar 
que la zona de captura aguas abajo del manantial es muy reducida comparada con el caso de un pozo. Por otro lado, una fuente de contaminación ubicada dentro de la zona de captura (Fig. 9, punto A) podría afectar la calidad del agua del manantial si la persistencia del contaminante es mayor que el tiempo de tránsito. Una fuente localizada de manera externa pero lateral al flujo de agua subterránea (Fig. 9, punto B) no tendría impacto sobre el manantial. En caso de localizarse aguas abajo del manantial (Fig. 9, punto C), la incidencia sería mínima.

\section{CONCLUSIONES Y RECOMENDACIONES}

El área de estudio presenta varios sectores con un potencial hidrogeológico sumado mayor de $5 \mathrm{~m}^{3} / \mathrm{s}$, producto de la elevada precipitación y la permeabilidad de las rocas, lo cual permitiría abastecer una población de 3 millones de habitantes con un consumo diario por persona de 150 litros.

Los manantiales que se presentan cercanos al río Virilla son producto de la descarga desde acuíferos en lavas fracturadas que reciben la recarga en las partes altas del macizo del volcán Barva por medio de precipitación que se infiltra y además reciben un aporte por medio de ríos influentes. En la hoja Barva y Naranjo la Falla de Alajuela influye en la ocurrencia de manantiales de importante caudal.

En la zona de estudio ocurren numerosos manantiales con caudales importantes que deben ser protegidos por medio de zonas de protección determinadas con base en tiempos de tránsito de bacterias y/o de agentes químicos y la zona más cercana al manantial debería ser de protección absoluta.

La simulación de la zona de captura de un manantial por medio de un modelo numérico, aún cuando se tenga una red de flujo como en el caso de "a" de la figura 2 , indica que es poco probable que un contaminante vertido aguas abajo del manantial alcance dicha fuente, pues esta zona es de descarga, la orientación de las líneas de flujo es preferencialmente ascendente y en la parte más baja del valle se origina un límite de no flujo que li- mita ambas zonas de descarga. Teóricamente no sería necesario establecer una zona de protección aguas abajo debido a las direcciones de flujo de contaminantes. No obstante lo anterior, las captaciones de agua de los manantiales se ubican por lo general aguas abajo, por lo que es importante definir una zona de protección para dichas captaciones.

Se propone aquí modificar la metodología que se utiliza para definir las zonas de protección de los pozos, de tal manera que la zona comprendida entre el punto de no retorno y el pozo (manantial) sea utilizada para proteger las captaciones de agua. En vista de que la zona de recarga aguas abajo para el caso de un manantial es menor que para el caso de pozos, se propone modificar las consideraciones legales estipuladas en la Ley Forestal, donde se establece un radio fijo alrededor del manantial. Se debería dar mayor énfasis a la zona de captura y definir una zona menor para las captaciones.

De la comparación de los diferentes métodos tanto manuales como matemáticos se concluye que la mejor propuesta para proteger un manantial consiste en definir la zona de captura con el método analítico o numérico y definir zonas de protección internas con base en los tiempos de tránsito de bacterias o de agentes contaminantes. Sobre esto último se deberían centrar de ahora en adelante las investigaciones, ensayando en diferentes tipos de suelos y rocas de la zona no saturada.

Sin embargo, la utilización del método analítico o numérico requiere de parámetros hidrodinámicos con los que en ocasiones no se cuenta, así como de personal calificado para manejar los programas de cómputo. Por esto en caso de no poder aplicar estos se propone la modificación a la Ley Forestal para definir una zona de radio fijo demarcando un círculo cuyo centro se encuentra a $175 \mathrm{~m}$ aguas arriba del manantial, dejando $25 \mathrm{~m}$ para las captaciones para darle mayor énfasis de protección a la zona de captura.

\section{REFERENCIAS}

ARREDONDO, S., 1994 a: Delimitación de las zonas de protección a los acuíferos del área de influencia del manantial de Ojo de Agua, San Antonio de Belén, 
Heredia. - 33 págs. Ser. Nac. de Aguas Subterráneas Riego y Avenamiento, San José [Inf. interno 187]

ARREDONDO, S., 1994 b: Delimitación de las zonas de protección a los acuíferos del área de influencia del manantial de Belén, San Antonio de Belén. - 21 págs. Serv. Nac. Aguas Subterráneas, San José [Inf. interno 188].

AyA., 2000: Aprendizaje del desarrollo humano. - 252 págs. Inst. Costarricense Acueductos y Alcantarillados, Dirección de obras rurales, San José.

BLANDFORD, T., HUYAKORN, P. \& SHU WU, Y., 1993: Well head protection delineation code. - 109 págs. IGMC, Colorado.

DARLING, W., PARKER, J., RODRÍGUEZ, H. \& LARDNER, A., 1984: Investigation of a volcanic aquifer system in Costa Rica using environmental isotopes. - Act. Sem. Reg. Empleo de Técnicas Isotópicas: 215-228, San José.

ECHANDI, E., 1981: Unidades volcánicas de la vertiente norte de la cuenca del río Virilla. - 123 págs. Univ. de Costa Rica, San José [Tesis Lic.].

FERNÁNDEZ, M., 1968: Datos generales sobre manantiales y brotes localizados durante los reconocimientos geo- lógicos de las partes norte y este de la cuenca del Río Virilla. - 14 págs. SENAS, San José [Inf. interno 54.]

FETTER, C. W., 1994: Applied hydrogeology. - 691 págs. [3ª ed.] Prentice, Nueva Jersey.

GÓMEZ, A., 1987: Evaluación del potencial de los acuíferos y diseño de las captaciones de agua subterránea en la zona de Puente de Mulas, provincia de Heredia, Costa Rica. - 23 págs. Univ. de Costa Rica, San José [Tesis Lic.].

LOSILLA, M., 1992: Mapa de fuentes de acueductos y sus áreas de recarga. - 17 págs. FUNDECOR, Heredia.

LOSILLA, M., ARIAS,G., CERVANTEZ, S. \& GÓMEZ, J., 1992: Estudio biofísico y socioeconómico del sector del cerro Guararí. - 51 págs. FUNDECOR, Heredia.

MCWHORTER, D. \& SUNADA, D., 1999: Groundwater hydrology and hydraulics. - 290 págs. [7 $7^{\mathrm{a}}$ ed.] Water Resources Publications, Washington.

MORA, M., 1995: Estudio geológico de un sector del Sureste de Grecia, Provincia de Alajuela. - 107 págs. Univ. de Costa Rica, San José [Inf. Campaña Geol.].

TODD, D.K., 1980: Ground water hydrology. - 336 págs. John Wiley, Nueva York. 